\title{
Dynamics of visual receptive fields in the macaque frontal eye field
}

2

5 1: Dept. of Neurobiology, Harvard Medical School, Boston, MA USA

6 2: Center for the Neural Basis of Cognition, Univ. of Pittsburgh, Pittsburgh, PA, USA

7 3: Dept. of Ophthalmology, Univ. of Pittsburgh, Pittsburgh, PA, USA

8 4: Dept. of Biomedical Engineering, Center for Cognitive Neuroscience,

9 and the Duke Institute for Brain Sciences, Duke University, Durham, NC, USA

10 5: Dept. of Bioengineering, Univ. of Pittsburgh, Pittsburgh, PA, USA

\#: Address correspondence to:

Matthew A. Smith

Department of Ophthalmology

University of Pittsburgh, Eye and Ear Institute

203 Lothrop St., $9^{\text {th }} \mathrm{FI}$.

Pittsburgh, PA, 15213

Tel: (412) 647-2313

Email: smithma@pitt.edu

Abbreviated title: Dynamics of receptive fields in FEF

Conflict of interest: "The authors declare no competing financial interests."

\section{Acknowledgements}

JPM was supported by NIH fellowship F32EY022529. MA Sommer was supported by NIH grant R01EY017592. MA Smith was supported by NIH grants R00EY018894, R01EY022928 and P30EY008098, a career development grant and an unrestricted award from Research to Prevent Blindness, and the Eye and Ear Foundation of Pittsburgh. We are grateful to R. Kelly, B. Yu, B. Cowley, H. Rao, and K. Bohon for helpful advice and discussion, and K. McCracken and R. Morrison for technical assistance. 


\section{Abstract}

Neuronal receptive fields (RFs) provide the foundation for understanding systems-level sensory processing. In early visual areas, investigators have mapped RFs in detail using stochastic stimuli and sophisticated analytical approaches. Much less is known about RFs in prefrontal cortex. Visual stimuli used for mapping RFs in prefrontal cortex tend to cover a small range of spatial and temporal parameters, making it difficult to understand their role in visual processing. To address these shortcomings, we implemented a generalized linear model (GLM) to measure the RFs of neurons in the macaque frontal eye field (FEF) in response to sparse, full-field stimuli. Our high-resolution, probabilistic approach tracked the evolution of RFs during passive fixation, and we validated our results against conventional measures. We found that FEF neurons exhibited a surprising level of sensitivity to stimuli presented as briefly as $10 \mathrm{~ms}$ or to multiple dots presented simultaneously, suggesting that FEF visual responses are more precise than previously appreciated. FEF RF spatial structures were largely maintained over time and between stimulus conditions. Our results demonstrate that the application of probabilistic RF mapping to FEF and similar association areas is an important tool for clarifying the neuronal mechanisms of cognition.

\section{Introduction}

The receptive fields (RFs) of frontal eye field (FEF) neurons are often mapped using reduced stimulus parameters outside the context of the main experimental task (Bruce and Goldberg 1985; Ferrera et al. 2009; Mayo and Sommer 2008; Phillips and Segraves 2010; Stanford et al. 2010; Thompson and Schall 1999). This conventional approach is generally restricted to localizing RF centers for subsequent stimulus presentation. Coarse maps of RFs seemed sufficient given the history of finding little visual selectivity in FEF visual responses (but see Barborica and Ferrera 2003; Mohler et al. 1973; Xiao et al. 2006) and the general hypothesis that the FEF and similar association areas, such as lateral intraparietal cortex, engage more in 
spatial operations_-"pointing" to locations for peri-saccadic vision or motor planning—than in featural analyses (e.g., Cavanagh et al. 2010; Mayo and Sommer 2010). But conventional RF mapping limits broader studies of visuomotor capabilities across FEF neurons such as RF interactions and task-dependent changes in RF sensitivity.

Our understanding of FEF RFs is therefore impoverished, except for some efforts to examine their spatial structure (Cavanaugh et al. 2012; Schall et al. 2004). One concern about the lack of detail is that it may contribute to misinterpretations of data. The study of presaccadic remapping provides one possible example. The RFs in FEF and other regions of visual cortex can shift their retinal positions just before a saccade (Duhamel et al. 1992; Sommer and Wurtz 2006; Umeno and Goldberg 1997). Such shifts are usually measured by characterizing RF centers, leaving open the possibility that apparent differences in RF dynamics across studies could stem from trivial experimental differences. For example, large RFs may appear to shift continuously, as opposed to jump discretely, because the "original" and "future" RFs overlap but could not be measured simultaneously. Thus, improved RF measures with better spatial precision could help to elucidate the role of FEF in vision.

Improvements in the temporal precision of FEF RF measurements are also needed. The majority of previous work on RF dynamics probed the RF location once or twice per trial (Joiner et al. 2013; Sommer and Wurtz 2006; Umeno and Goldberg 1997; Zirnsak et al. 2014). Even though some studies used brief stimuli, around 25-50 ms in duration, a more complete understanding of active vision may require temporally precise knowledge about the dynamics of neuronal responses to multiple, repeated stimuli. However, it is unclear if FEF visual responses maintain their fidelity at fast stimulus presentation rates. More precise measures of temporal sensitivity would therefore help clarify the role of FEF in dynamic cognitive processing.

As a first step in characterizing FEF RFs in detail, we focused on their spatiotemporal organization during steady fixation. We mapped the extent of FEF RFs with high temporal precision using sparse, stochastic dot stimuli. We used a probabilistic approach to measure the 
relationship between visual stimuli and spiking activity in FEF neurons using a generalized

87 linear model (GLM) (Kelly et al. 2010; Pillow et al. 2008). Probabilistic mapping provided a richer measure of FEF RFs from a comparable amount of data obtained using conventional methods. FEF neurons had reliable responses to repeated stochastic dots shown as briefly as $10 \mathrm{~ms}$ if only one or a few dots were present on the screen simultaneously. Our findings characterize the time course of visual sensitivity in FEF neurons and provide a foundation for studying the neuronal circuitry underlying stable visual perception and motor planning during natural behavior.

\section{Materials and Methods}

Surgical Preparation. Two adult rhesus macaques, one female and one male (Macaca mulatta; monkeys $\mathrm{L}$ and $\mathrm{U}$ ), were surgically implanted with headposts and an FEF recording chamber (stereotaxic coordinates: 25 anterior, 20 lateral) using sterile surgical techniques under isoflurane anesthesia. All procedures were approved by the Institutional Animal Care and Use Committee of the University of Pittsburgh and complied with guidelines set forth in the National Institute of Health's Guide for the Care and Use of Laboratory Animals.

\section{Experimental Setup. Stimuli were displayed on a 21" CRT monitor with a resolution of} $1024 \times 768$ / 768x384 pixels and a refresh rate of $60 / 100 \mathrm{~Hz}$ at a distance of $36 / 43 \mathrm{~cm}$ (Monkeys $\mathrm{L} / \mathrm{U})$. All stimuli were white dots $\left(104 \mathrm{~cd} / \mathrm{m}^{2}\right)$ on a black background $\left(0.0 \mathrm{~cd} / \mathrm{m}^{2}\right)$. Stimuli were generated using the REX real-time system (Hays et al. 1982) for Monkey $L$ and custom software written in Matlab (Mathworks, Natick, MA) including Psychophysics toolbox extensions (Brainard 1997; Kleiner 2007) for Monkey U. Stimulus timing and alignment was confirmed using a photodiode. Eye position was monitored via infrared eye tracking (Monkey L: ISCAN, Bowdoin, MA; Monkey U: EyeLink 1000, SR Research, Mississauga, Canada). We acquired data with the 
111 REX real-time system for Monkey $L$ and a Grapevine recording system (Ripple; Salt Lake City,

112 UT) for Monkey U.

114 Electrophysiological methods. Extracellular recordings were made with tungsten

115 microelectrodes (300 k $\Omega$ to $1 \mathrm{M} \Omega$ impedance @ $1 \mathrm{kHz} ; \mathrm{FHC}$, Bowdoinham, ME) advanced with a 116 NIH-designed mechanical microdrive (Laboratory for Sensorimotor Research, Bethesda, MA).

117 Recordings were aligned using a plastic grid placed inside the recording chamber (Crist

118 Instruments, Hagerstown, MD). FEF was visually identified on the anterior bank of the arcuate

119 sulcus and physiologically verified as the chamber grid holes where we could evoke fixed-vector

120 saccades reliably ( $>50 \%$ of the time) using low current ( $\leq 50 \mu \mathrm{A}$ for $70 \mathrm{~ms}$ ) microstimulation

121 (Bruce et al. 1985) and grid holes immediately adjacent to those sites. Isolation of single

122 neurons was performed online using time-amplitude window discrimination and later refined

123 offline using custom software in Matlab (Kelly et al. 2007). We typically recorded one isolated

124 neuron per recording session, but occasionally isolated two units.

125 Conventional RF mapping. The conventional RF of each neuron was mapped using a memory126 guided saccade (MGS) task (Hikosaka and Wurtz 1983). The trial began when the monkey 127 fixated a central blue circle $\left(0.5^{\circ}\right.$ diameter $)$. After maintaining fixation within $1.8^{\circ}$ for $200 \mathrm{~ms}$, a 128 peripheral target $\left(1.8^{\circ}\right.$ diameter) was flashed for $50 \mathrm{~ms}$ at one of eight locations (cardinal and 129 orthogonal directions, typically $10^{\circ}$ from fixation). The animal was required to maintain fixation 130 for another 250 or $400 \mathrm{~ms}$ until the fixation point was extinguished. If the animal made a 131 saccade to the previously cued (remembered) location within $200 \mathrm{~ms}$ and maintained gaze 132 within $2.7^{\circ}$ of that location for $150 \mathrm{~ms}$, it received a liquid reward. One block consisted of 133 pseudorandomized presentations of all eight directions, and typically ten blocks were acquired 134 per neuron. Visually-guided saccades (VGS) were occasionally used instead of the MGS task to 135 map receptive fields. 
137 Characterization of classical RFs. The location of each neuron's classical RF was determined 138 using vector averaging of neuronal responses across radial stimulus positions in the MGS or 139 VGS task. A tuning bias vector was calculated (Leventhal et al. 1995) to determine the direction 140 of the conventional RF relative to fixation (black triangle, Fig. 1A). Mean visual responses at a 141 range of amplitudes (e.g., 3-30 deg.) during a second series of saccades at or near the 142 preferred direction were fitted with a Gaussian function (Matlab function: Isqcurvefit). The peak 143 of that function defined the best amplitude for a given neuron.

145 Visuomotor and sustained-transient indices. Following identification of the direction and 146 amplitude of the RF, we used the same responses to determine the relative strength of a 147 neuron's visual and motor components. The visual response was defined as 50-150 ms after 148 stimulus onset, and the motor response was defined as -50 to $50 \mathrm{~ms}$ around saccade onset. 149 Baseline firing rate was also calculated from the $100 \mathrm{~ms}$ preceding stimulus onset. The number 150 of spikes within each epoch across all directions was used to calculate a visuomotor index (VMI) 151 for each neuron ([visual - motor] / [visual + motor]; (Bruce and Goldberg 1985). VMI ranges 152 from -1 for neurons with purely movement responses to 1 for neurons with purely visual 153 responses.

A similar procedure was used to estimate the relative amount of sustained and transient 155 activity to produce a transient-sustained index (TSI) per neuron. We defined the transient 156 component as the firing rate $0-50 \mathrm{~ms}$ after the time to half of the maximum of the initial visual 157 response and the sustained component as the firing rate 50-100 ms after time to half max., 158 where ([transient - sustained] / [transient + sustained]). TSI ranged from -1 for neurons with 159 only sustained responses to 1 for neurons with only transient responses. 
A signal-to-noise ratio (SNR) was calculated for units recorded on the Ripple system to

estimate neuronal isolation. The SNR was determined as the ratio of the amplitude of the average waveform to the standard deviation of the noise (Kelly et al. 2007).

Probabilistic RF mapping. After conventional RF mapping, we used stochastic dot stimuli to probabilistically map the same RF. Animals were trained to fixate on a central blue circle ( $3 \mathrm{sec}$ for Monkey U; 2 sec for Monkey L). During this time, white square dots were presented at random locations over the entire screen, and each dot was scaled to increase in size with eccentricity to approximately match the magnification factor in V1 RFs (Dow et al. 1981). At the extremes, a dot shown at fixation was $\sim 0.16^{\circ}$ in diameter and a dot at the rightward edge of the screen at the vertical midpoint $\left(\sim 28^{\circ}\right.$ eccentric) was $\sim 0.95^{\circ}$ in diameter. A single trial consisted of many dot stimulus images that were refreshed many times. The passive fixation task reduced the influence of saccade planning on neuronal activity and increased the number of possible stimulation presentations per trial. If the monkey broke fixation or made a saccade to one of the RF-probing stimuli, the trial was aborted and no reward given. Dots were flashed briefly (10-167 ms per image), and animals quickly learned to not respond to them. All analyses were performed on correctly completed trials.

To probe the spatial and temporal sensitivity of FEF neurons, we varied 1) the number of dots presented per stimulus image ("dots task"), or 2) the duration of each stimulus image per frame ("dwell task"). For Monkey U, we varied parameters across randomly interleaved trials in one of three ways: one, two, or eight dots per image at $70 \mathrm{~ms}$ image duration; one dot per image at 30,70 , or $150 \mathrm{~ms}$ image durations; and one dot per image at 10 or $70 \mathrm{~ms}$ image durations. For Monkey L, we used one dot per image at $167 \mathrm{~ms}$ image duration. In all cases, the number of dots per image and the image durations were fixed within a trial. We varied at most one parameter (number of dots or image duration) per recording session. We recorded $535 \pm 38$ SEM (range: 96-1590) trials per condition per session. Because of issues inherent in 
maintaining neuronal isolation, we were not able to record conventional and probabilistic RFs for every neuron; for this reason, we provide the sample sizes in Figures 2, 4, and 5.

\section{Generalized linear model for probabilistic RF mapping. For each neuron, we formulated a} generalized linear model (GLM) to predict the spiking activity $(Y)$ as a linear combination of the pixel values $(X)$ plus noise: $Y=X \beta+e$, where $\beta$ are the coefficients or weights fit by the GLM and e is an error term. For computational expediency, we downsampled the screen by a factor of 32 to create "super-pixels", each $\sim 1.9$ deg. square. Each super-pixel was assigned a value of 1 if it contained an illuminated pixel in the full image, and 0 if it did not. A value between 0 and 1 was assigned if a dot stimulus fell on the border, based on the proportion of its area that in each super-pixel. This process resulted in a 32x24 element matrix (a 768-element vector) for each dot image shown in experiments with Monkey $U$. We used a $24 \times 16$ element matrix and a 384element vector for Monkey L, but the analysis was otherwise identical. This vector became a row in the design matrix $\mathrm{X}$, and the number of rows in $\mathrm{X}$ was determined by the number of dot images shown (concatenated across fixation trials). For each row in X, we counted spikes occurring within a time window of $\Delta t \mathrm{~ms}$ to $\Delta t+d \mathrm{~ms}$ relative to the dot image onset. $\Delta t$ and $d$ account for the visual onset latency and the duration of the impulse response for each neuron. Solving the GLM produces a coefficient matrix $\beta$ with 768 values representing the coefficients that predict spiking from the visual stimulus at each super-pixel's location on the screen - a “probabilistic RF” (gImRF).

GLM fits were obtained using the "glmnet" toolbox in Matlab (Friedman et al. 2010). We set the maximum of the non-zero lambdas to $20 \%$ of the pixel values and used an alpha value of 1 (lasso regression, a L1-norm). Our results were not sensitive to the particular parameters of the GLM within a wide range of options and ridge regression (L2-norm) produced qualitatively similar results. 
We used five-fold cross-validation to compare GLMs across conditions. Additionally,

212 conditions with faster frame rates (e.g., 10 and $30 \mathrm{~ms}$ per image) consisted of more stimulus

213 presentations than slower frame rate conditions (e.g., $150 \mathrm{~ms}$ per image). The difference in

214 total stimuli presented in each condition could lead to differences in the quality of GLM fits

215 between conditions, so we subsampled the number of trials in the dwell task such that the total

216 number of stimulus presentations was equal across conditions. For non-overlapping sets of

$21780 \%$ of these trials, we obtained a GLM fit. For each of these five sets of $\beta$ values, we

218 evaluated the GLM's fit equation to produce estimated spike trains in response to images from

219 the training trials in all conditions. In this way, we could ensure that GLMs were not specific to

220 the conditions in which they were built. For each model and cross-validation iteration, we

221 computed the Pearson's correlation between the predicted and observed spike counts, giving us

222 a correlation value $(r)$ and $p$-value. The mean of these five values was used to evaluate the

223 predictive power of the RF model for each cell in each condition (see Statistical evaluation

224 below).

225 To estimate the ideal spike window latency and duration for each neuron, we first fit the

226 spiking activity with 624 different GLMs (26 latencies, $0-250 \mathrm{~ms}$ in $10 \mathrm{~ms}$ steps $\times 24$ durations,

$227 \quad 10-250$ in 10 ms steps) for each condition, spanning the range of plausible FEF response

228 latencies and durations (Mayo and Sommer 2013). The 624 GLMs were fit to the equivalently-

229 sampled data. A 2D Gaussian was fit to the resulting GLM matrix (Fig. 3C) and the location of

230 its peak was selected as the optimal spike window latency and duration. Cross-validation was

231 always used when comparing GLMs between conditions or with different spike alignments.

232 Once the optimal condition and spike window were chosen, we fit a 2D Gaussian to the GLM

233 built on all trials with those parameters to determine the glmRF position and size.

235 Statistical evaluation of probabilistic RFs. For each spatial $\beta$ map, we used a resampling test to 236 determine significance. First, we calculated the mean and standard deviation of the $\beta$ values 
and found the area of the largest contiguous set of $\beta$ values that were two standard deviations away from the mean. Next, we randomly shuffled the $\beta$ values 1000 times and recalculated the largest contiguous area each time. If the area from the original, unshuffled map was at least three super-pixels (2.7 square deg./super-pixel) large and fell outside the $99 \%$ confidence interval of the shuffled map values, we declared that map as having a significant glmRF. We

242 included any neuron with a significant gImRF on any $\beta$ map in further analyses. Three neurons 243 had significant inhibitory RFs without accompanying excitatory RFs, and we did not correct for 244 RFs on the edge of the screen (see elongated RFs in the periphery in Fig. 2D). An identical 245 resampling procedure was used to determine the significance of cross-validation by resampling 246 the contiguous, significant $p$-values $(p<0.01)$ of the Pearson's r-values in the GLM matrices 247 (Fig. 3C). We also included neurons that exhibited clear glmRFs by eye and yielded a significant glmRF after resampling all trials, but failed cross-validation $(n=28)$. These files tended to have fewer trials overall. We found no difference between the cross-validated and "significant by resampling but not cross-validated" cells, but for clarity we represent each population by different symbols in Figures 2 and 5 .

\section{Results}

254 We recorded from 186 neurons in FEF in two monkeys (26 from Monkey L). We mapped the 255 RFs of FEF neurons using both conventional and GLM-based methods, which yielded the 256 conventional RF and gImRF for each neuron. Mapping RFs and glmRFs allowed us to validate 257 our approach, quantify the accuracy of glmRFs, and measure the relatively stable RF dynamics 258 in FEF.

RFs were mapped conventionally using visually- or memory-guided saccades. Visual responses to stimuli presented during conventional mapping yielded RF estimates such as

261 those in Figure 1. This neuron's RF was located up and to the right (Fig. 1A) and most 262 responsive $\sim 15$ degrees from the fixation point (Fig. 1B). 
To address the limitations of conventional mapping, we also measured FEF RFs using

264 probabilistic mapping, which samples the full visual field in an unbiased manner. While the

265 monkey fixated on a central target, they were presented with visual stimuli at pseudorandom

266 locations and rewarded for maintaining fixation. Monkeys learned to ignore the peripheral

267 stimuli within a single training session. In contrast to conventional mapping which tests a single

268 location per trial, probabilistic mapping permitted us to test dozens to hundreds of stimulus

269 locations per trial. We varied the number of stimuli or the stimulus presentation rate during a

270 recording session to measure the spatial or temporal sensitivity of FEF neurons. Neuronal

271 responses were analyzed as a function of visual stimuli in the sequential video frames using a

272 GLM (with a $L_{1}$ norm for regularization to produce a sparse model) and statistical analyses (Fig.

$2731 \mathrm{C})$ to generate the glmRF in space and time. For the same example neuron, we used

274 conventional RF mapping (Fig. 1A,B), as well as probabilistic mapping using stimulus frames

275 presented every $70 \mathrm{~ms}$ during trials consisting of three seconds of fixation. The resulting glmRF

276 (Fig. 1D) matched the conventionally obtained RF well, but provided much more detail.

neurons are summarized in Figure 2A. We ran the GLM analysis on all 186 FEF neurons and measuring the angular difference relative to fixation (Fig. 2B). The distribution of differences was not significantly different from zero $\left(8.3^{\circ} \pm 7.4^{\circ}\right.$, t-test, $\left.p=0.27\right)$; more generally, $72 \%$ of the neurons' RFs and glmRFs were within 45 degrees of each other, and $47 \%$ were within 20

287 degrees. We compared this distribution against a bootstrap data set composed of randomly 288 paired RF and glmRF data from different neurons, and found that the angular differences we 
observed were significantly smaller than the chance expectation from this bootstrap test $(p<$ 0.001).

The eccentricities of RFs and glmRFs for each neuron were likewise similar (Fig. 2C).

292 Conventional RF mapping included large amplitude saccades (20-40 deg.) across the full extent 293 of the monitor, while probabilistic mapping used fixation in the center of the screen.

294 Conventional mapping therefore tested a larger range of eccentricities than probabilistic 295 mapping and may have led us to underestimate the eccentricity of RFs that overlapped the 296 edge of the display. We fit the conventional RF mapping data with a log Gaussian (Bruce and 297 Goldberg 1985). RF and gImRF amplitudes were highly correlated (Spearman's rho $=0.61, p<$ 298 0.001), but conventional RF amplitudes were significantly larger (signed-rank test, $p=0.002$ ) . More importantly, probabilistic mapping yielded RFs that were highly consistent with those determined by conventional methods while far exceeding their spatial and temporal resolution.

Given the consistency between conventional and probabilistic RFs, the remaining analyses focus on the more spatially detailed glmRFs. For each neuron, we selected the spike window parameters that provided the best description of its glmRF. The largest area of significant, contiguous screen pixels defined the glmRFs and was fit with a 2D Gaussian. (Better fits may be achievable with a more complex mixture of Gaussians; this is an avenue for future research.) We recorded from both hemispheres, and glmRFs consequently spanned the full visual field (Fig. 2D, each ellipse drawn with a radius equal to one standard deviation of the 2D Gaussian fit). With one exception, glmRFs were centered in the visual field contralateral to 309 the recording hemisphere. We used the ellipses in Fig. 2D as an estimate of the area of each 310 glmRF. As in other parts of visual cortex and consistent with previous reports (Cavanaugh et al. 311 2012), glmRFs increased in size as they became more eccentric (Fig. 2E). The relationship 312 between the size of the glmRF and its eccentricity was, as expected, even more pronounced for 313 the best fit glmRFs (Fig. 2E, black symbols and line). Thus, the location, eccentricity, and 314 extent of glmRFs are an excellent match to conventionally-derived measures. 
We explored the possibility that probabilistic mapping could be biased to yield glmRFs for neurons with certain firing characteristics. We computed four metrics for each neuron (mean

$317 \pm$ SEM): 1) its baseline firing rate (17.2 \pm 1.9 spikes/sec); 2$)$ the relative strength of its visual-

318 and saccade-related responses, as measured by the visuomotor index (VMI: $0.13 \pm 0.05$ ); 3 ) the

319 relative strength of its transient and sustained response components, as measured by the

320 transient-sustained index (TSI: $0.2 \pm 0.03$ ); and 4) the quality of single-neuron isolation, as

321 quantified by the recording's single-to-noise ratio (SNR: $3.02 \pm 0.14$ ). We found no significant

322 correlations between these four metrics and the glmRF goodness-of-fit (Spearman's rho values

$323=0.15,0.07,-0.12,0.12 ; p>0.2$ for all four correlations). We expected more visually-

responsive neurons $(\mathrm{VMI} \approx 1)$ to yield better glmRFs, but this was not the case. We suspect that

325 this is because the VMI is derived from a saccade task and therefore more susceptible to

326 preparatory motor activity than the passive fixation task, and therefore a less sensitive measure

327 of visual responsiveness. Overall, probabilistic mapping was relatively unbiased in its ability to

328 reveal glmRFs in terms of these four distinguishing features.

Probabilistic mapping allows for viewing RF changes over time, and such measurements of a neuron's "temporal impulse" are essential for determining if FEF neurons can track stimulus changes on a fine timescale. This issue is related to but distinct from most previous work that measured the responses of FEF neurons to a single brief stimulus presentation. Above, we selected the optimal conditions for each neuron that yielded the best glmRF. To determine the

334 shape of the temporal impulse, we can also shift the spiking activity relative to the time of 335 stimulus presentations to understand the temporal sensitivity of an individual neuron. Figure 3A 336 illustrates the evolution of glmRFs for three neurons each in a $270 \mathrm{~ms}$ epoch surrounding the 337 optimal response. For this analysis all of the image and spiking data were fed into a single GLM run across all temporal offsets, such that the betas were only fit once for the entire temporal kernel. The left column illustrates 9 frames of a glmRF measured at $30 \mathrm{~ms}$ resolution, while the right two columns illustrate 27 frames each of glmRFs measured at a 10 ms resolution. In all 
341

342

cases, there is a smooth transition from no relationship between spikes and stimuli (green), to a punctate, ellipsoidal ideal alignment (red), and back again to baseline (green). An important commonality is that all of them show a surprisingly strong and spatially consistent response, even though the stimuli that evoked the responses were brief, rapidly flashed, and stochastic. The duration of each glmRF clearly differed between individual neurons (optimal spike window duration: $118 \pm 5.5 \mathrm{~ms}$, mean \pm SEM; range: $30-190 \mathrm{~ms} ; \mathrm{n}=63$ ). The dynamics of the glmRFs in Figure 3A are summarized by their temporal kernels in Figure 3B. Temporal kernels are created by sliding a fixed-duration spike window over a range of spiking latencies and visual stimulus offsets. They capture changes in visual sensitivity over time, centered on the best-fit spike-stimulus alignment, and summarize each frame's spatial variance (Yeh et al. 2009). Onedimensional temporal kernels can be expanded to 2-D temporal matrices by allowing the spike window duration to also vary (Fig. $3 \mathrm{C})$. In our analyses, the temporal matrix of a cell covers a broad range of spike window delays and durations and represents the results for 624 independent GLMs per neuron (26 latencies x 24 durations). Correlations between observed and model-predicted spike trains were relatively weak when the spike window was short and immediately followed stimulus onset (bottom left corners of matrices). As the spike window increased in duration, the GLM's spiking activity was a better match to the actual spike trains (transition from blue to red along left vertical axis). The combination of frame-by-frame plots of visual space (Fig. 3A) and broader descriptions of temporal sensitivity (Fig. 3B, C) illustrate that the three representative neurons responded well to visual stimuli presented every 10-30 ms.

Because temporal sensitivity varied across individual neurons, we again asked if factors such as the degree of visual responsiveness or neuronal isolation could account for the variability. We found no significant correlations between spike window latency or duration with VMI, SNR, or baseline firing rate (all comparisons $p>0.1$ ). However, we found a significant correlation between TSI and spike window duration such that neurons with more sustained responses $(\mathrm{TSI}<0)$ were associated with longer spike windows (Spearman's rho $=-0.29, p<$ 
0.05). This confirms that our analysis appropriately adapted to the firing characteristics of individual neurons. Also, spike window latency and width were each significantly correlated with the goodness-of-fit of glmRFs across neurons (rho $=-0.39$ and rho $=0.48$, respectively; both $p<$ $0.001)$, although not with each other $(p=0.68)$, such that neurons with relatively short-latency responses and a longer response to each dot image tended to yield the best-fit glmRFs. Independent populations of FEF neurons, recorded using different stimulus presentation 373 rates, showed similarly broad temporal sensitivity as stimulus-spike alignment was shifted away 374 from optimal. The population-averaged temporal kernels for neurons in conditions with 30 and 375 10-ms per stimulus are shown in Figure 4A (blue and red, respectively). Regardless of the 376 stimulus presentation rate, FEF neurons were roughly half as responsive when using their 377 optimal spike window durations and offsetting the spike-stimulus alignment by $30-45 \mathrm{~ms}$. These 378 results suggest that spatiotemporal integration may be relatively brief for visually-responsive 379 FEF neurons.

The population-averaged temporal matrix is shown in Figure 4B is made by averaging 381 the normalized matrices from each cell. The peak predictive value of our glmRFs, when 382 assayed across the entire population, corresponded to a window delay of roughly $50 \mathrm{~ms}$ with a 383 window width of roughly $125 \mathrm{~ms}$. These values correspond to previous estimates of FEF latency 384 and response duration to a brief flash (Mayo and Sommer 2013; Pouget et al. 2005). More 385 importantly, the smooth variation of this population average indicates that our glmRFs were able 386 to predict spikes well when the temporal kernel of each cell was considered, and predictive 387 power fell off rapidly as the spikes and images were poorly aligned. Although FEF neurons clearly responded to sparse stimuli at rapid presentation rates 389 (Figs. 3-4), their responses only marginally improved, if at all, when using slower frame rates or 390 more visual stimuli. To illustrate this point, for each neuron we averaged the beta values within 391 a 5-pixel radius centered on the optimal spike window for each condition. (Results were similar 392 for radii 1-10). We then normalized each neuron to its average beta across conditions so that 
the relative sensitivity to image durations/number of dots could be visualized by its deviation

394 from 1. Figure 5 shows representative single neurons for each combination of stimulus

395 presentation rates (Fig. 5A,B) and number of dots (Fig. 5C) tested. Population results are

396 illustrated in Figures 5D-F. Despite the trend for preferring longer image durations,

397 spatiotemporal tuning was not significantly different across conditions in each of the three sub-

398 tasks (one-way ANOVA, $p>0.13$ in all tasks). Differences in optimal spike window latency and

399 duration across conditions for the population were likewise not significant (one-way ANOVA, p >

$400 \quad 0.16$ in all tasks for both latency and duration). It is important to note here that because of the

401 subsampling of image presentations at shorter image durations (see Methods), glmRFs with

402 equivalent predictive power were therefore acquired more rapidly at faster stimulus presentation

403 rates (i.e., it took $20 \%$ of the time to acquire the glmRFs at $30 \mathrm{~ms}$ compared to $150 \mathrm{~ms}$ ). Thus,

404 the goal of acquiring the "best" RF per unit time (i.e., most predictive of spiking response) was

405 best served with faster stimulus presentation, even if the trend was slightly in the opposite

406 direction in Figure 5D and $\mathrm{E}$.

407 We did see spatial interactions in a few single neurons, such as evidence for a

408 suppressive surround based on negative beta values outside the excitatory RF. Spatial

409 integration in FEF has been explored previously in FEF (Cavanaugh et al. 2012; Schall et al.

410 2004). Given that our sample of neurons varying the number of dots was small $(n=23)$, we did

411 not explore this issue in detail. Initial measurements of dot interactions over time (e.g., the

412 distance between dots on consecutive frames) and spiking activity also did not yield notable

413 effects. However, our method of mapping RFs using stochastic stimuli could easily be adapted

414 to test specific predictions about spatial integration in FEF.

416 Discussion

417 We measured the receptive fields of visually-responsive FEF neurons using sparse noise stimuli 418 and regressed the spike trains and images using a GLM (Fig. 1). Independent measures of 
probabilistic and conventional RFs produced well-matched estimates of RF locations,

420 eccentricities, and sizes (Fig. 2). While conventional RF mapping remains a useful way of

421 estimating RF centers, probabilistic mapping provides a richer estimate of the RF's extent, sub-

422 structure, and changes in those features over time (Fig. 3). We used brief, task-irrelevant

423 stimuli. Nonetheless, we found that FEF neurons responded robustly and consistently to sparse

424 dot stimuli presented as briefly as every $10 \mathrm{~ms}$ and to many dots on the screen simultaneously

425 (Fig. 4-5).

Stochastic approaches to measuring RFs have been widely implemented throughout the

427 visual system, ranging from linear spike-triggered averaging approaches (Churan et al. 2012 in

428 SC; Jones and Palmer 1987 in V1; Livingstone et al. 2001 in MT; Reid and Alonso 1995 in LGN;

429 Sakai and Naka 1987 in retina) to more sophisticated methods for recovering nonlinear

430 neuronal RFs such as spike-triggered covariance (Rust et al. 2005) and nonlinear RF models

431 (David et al. 2004). Recently, a few studies have employed generalized linear models as a

432 means of probabilistically determining neuronal responses based on the visual stimulus

433 (Calabrese et al. 2011; Hartmann et al. 2011; Kelly et al. 2010; Park et al. 2014; Pillow et al.

434 2008). These models are convenient because they naturally fit into a statistical framework,

435 allowing for simple assessment of the RF model's significance and implementation of methods

436 like regularization to constrain model outcomes. They are also easily extensible to incorporate

437 additional signals that may influence neuronal activity, such as the activity of nearby neurons

438 (Kelly et al. 2010; Pillow et al. 2008) or specific metrics extracted from the visual stimulus

439 (Fernandes et al. 2014). Finally, the usefulness of probabilistic approaches will only increase as

440 computational resources advance, for example, obviating downsampled visual stimuli and

441 allowing more model parameters to be tested simultaneously.

442 Despite the potential advantage of probabilistic approaches to measure neuronal RFs,

443 they have been rarely utilized in prefrontal cortex. Coarser RF measures have been sufficient

444 for a number of important studies of FEF's role in oculomotor control, attention, and visual 
stability (Noudoost et al. 2010; Schall 2004; Sommer and Wurtz 2008). A complete understanding of the dynamic nature of vision, however, will require increased spatial and temporal sensitivity. A prime example of this comes from recent contrasting results in spatial RF dynamics around the time of a saccade (Sommer and Wurtz 2006; Wurtz 2008; Zirnsak et al. 2014). The application of probabilistic approaches to higher-order cortical neurons, along with expansion beyond passive fixation into more detailed experimental tasks, will permit the study of RF dynamics in more naturalistic contexts.

We found that FEF contains neurons with surprisingly exquisite sensitivity to visual stimuli, roughly an order of magnitude more temporally precise than previous estimates. Specifically, Figure 5D-F suggests that optimal data collection (i.e., minimizing reward rates and maximizing RF sampling), given the parameter ranges that we tested, can be achieved using eight stimuli, each $0.16^{\circ}$ in size when near fixation (see Methods), simultaneously and stochastically presented every 10-30 ms. We used an L1-norm, lasso regression to normalize our GLM model, tested a range of spike window latencies and durations, and downsampled the raw pixel data for computational expediency, but these modifications were not necessary to obtain viable data. Our results are consistent with FEF's role as a visual area, driven by visual inputs from the superior colliculus and extrastriate visual cortex (Schall et al. 1995). Perisaccadic vision and mechanisms of visual stability likely require high precision because eye movements cause frequent disruptions of visual input. Our results add to the growing literature on the role of FEF in rapid and temporally precise cognitive processing (Stanford et al. 2010). Improvements in the measurement of RF dynamics can help resolve longstanding issues regarding peri-saccadic vision. Indeed, previous work shows that coarse RF estimates can hide the fine dynamics of RFs, possibly obscuring what happens peri-saccadically (Sommer and Wurtz 2006; Zirnsak et al. 2014). Increasing the spatial and temporal precision of RF measurements has proved fruitful in elucidating circuits in number of settings: the computation of pattern motion selectivity in area MT (Pack and Born 2001; Smith et al. 2005), understanding 
471 suppressive phenomena in V1 (Bair et al. 2002; Smith et al. 2006; Smith et al. 2007), and the

472 dynamic shifts of RFs with attention in MT (Anton-Erxleben et al. 2009; Womelsdorf et al. 2006).

473 The prefrontal cortex has been notably absent from these types of advances in RF

474 measurement, despite the need for such precision in measuring RF dynamics during the brief

475 and turbulent peri-saccadic period. The detail provided by increasing the spatial and temporal

476 resolution of RF measurements around the time of an eye movement is critical to revealing the

477 neural circuitry of peri-saccadic vision.

$478 \quad$ Taken together, our results highlight two previously unreported aspects of the responses

479 of FEF neurons: (1) rapid temporal integration and (2) reliable responses to repeated, briefly

480 presented stimuli that are behaviorally irrelevant. Although FEF neurons varied in their

481 responses to our stochastic stimuli, as a population there was no evidence that these findings

482 were confined to a specialized FEF population. Instead this appears to represent the profile of

483 visually-responsive FEF neurons. These findings suggest a reappraisal of the conventional

484 view of FEF and demonstrate new experimental approaches to test the role of FEF neurons in 485 active vision. 
Anton-Erxleben K, Stephan VM, and Treue S. Attention Reshapes Center-Surround Receptive Field Structure in Macaque Cortical Area MT. Cereb Cortex 19: 2466-2478, 2009. Bair W, Cavanaugh JR, Smith MA, and Movshon JA. The Timing of Response Onset and Offset in Macaque Visual Neurons. J Neurosci 22: 3189-3205, 2002.

492 Barborica A, and Ferrera VP. Estimating invisible target speed from neuronal activity in 493 monkey frontal eye field. Nat Neurosci 6: 66-74, 2003.

494 Brainard DH. The Psychophysics Toolbox. Spat Vis 10: 433-436, 1997.

495 Bruce CJ, and Goldberg ME. Primate frontal eye fields. I. Single neurons discharging before 496 saccades. J Neurophysiol 53: 603-635, 1985.

497 Bruce CJ, Goldberg ME, Bushnell MC, and Stanton GB. Primate frontal eye fields. II.

498 Physiological and anatomical correlates of electrically evoked eye movements. J Neurophysiol 499 54: 714-734, 1985.

500 Calabrese A, Schumacher JW, Schneider DM, Paninski L, and Woolley SMN. A

501 Generalized Linear Model for Estimating Spectrotemporal Receptive Fields from Responses to 502 Natural Sounds. PloS one 6: e16104, 2011.

503 Cavanagh P, Hunt AR, Afraz A, and Rolfs M. Visual stability based on remapping of attention 504 pointers. Trends Cogn Sci 14: 147-153, 2010.

505 Cavanaugh J, Joiner WM, and Wurtz RH. Suppressive surrounds of receptive fields in 506 monkey frontal eye field. J Neurosci 32: 12284-12293, 2012.

507 Churan J, Guitton D, and Pack CC. Spatiotemporal structure of visual receptive fields in 508 macaque superior colliculus. 2012, p. 2653-2667.

509 David SV, Vinje WE, and Gallant JL. Natural Stimulus Statistics Alter the Receptive Field 510 Structure of V1 Neurons. The Journal of Neuroscience 24: 6991-7006, 2004.

511 Dow BM, Snyder AZ, Vautin RG, and Bauer R. Magnification factor and receptive field size in 512 foveal striate cortex of the monkey. Exp Brain Res 44: 213-228, 1981.

513 Duhamel JR, Colby CL, and Goldberg ME. The updating of the representation of visual space 514 in parietal cortex by intended eye movements. Science 255: 90-92, 1992.

515 Fernandes HL, Stevenson IH, Phillips AN, Segraves MA, and Kording KP. Saliency and 516 Saccade Encoding in the Frontal Eye Field During Natural Scene Search. Cereb Cortex 24: 517 3232-3245, 2014.

518 Ferrera VP, Yanike M, and Cassanello C. Frontal eye field neurons signal changes in decision 519 criteria. Nat Neurosci 12: 1458-1462, 2009. 
Friedman J, Hastie T, and Tibshirani R. Regularization Paths for Generalized Linear Models via Coordinate Descent. Journal of statistical software 33: 1-22, 2010.

Hartmann TS, Bremmer F, Albright TD, and Krekelberg B. Receptive field positions in area MT during slow eye movements. J Neurosci 31: 10437-10444, 2011.

Hays AV, Richmond BJ, and Optican L. A UNIX-based multiple process system for real-time data acquisition and control. WESCON Conf Proc 2: 1-10, 1982.

Hikosaka O, and Wurtz RH. Visual and oculomotor functions of monkey substantia nigra pars reticulata. III. Memory-contingent visual and saccade responses. J Neurophysiol 49: 1268-1284, 1983.

Joiner WM, Cavanaugh J, and Wurtz RH. Compression and suppression of shifting receptive field activity in frontal eye field neurons. J Neurosci 33: 18259-18269, 2013.

Jones JP, and Palmer LA. The two-dimensional spatial structure of simple receptive fields in cat striate cortex. 1987, p. 1187-1211.

Kelly RC, Kass RE, Smith MA, and Lee TS. Accounting for network effects in neuronal responses using L1 regularized point process models. Advances in neural information processing systems 23: 1099-1107, 2010.

\section{Kelly RC, Smith MA, Samonds JM, Kohn A, Bonds AB, Movshon JA, and Sing Lee T.} Comparison of Recordings from Microelectrode Arrays and Single Electrodes in the Visual Cortex. The Journal of Neuroscience 27: 261-264, 2007.

Kleiner M, Brainard, D., Pelli, D. What's new in Psychtoolbox-3? Perception 36: 2007. Leventhal A, Thompson K, Liu D, Zhou Y, and Ault S. Concomitant sensitivity to orientation, direction, and color of cells in layers 2, 3, and 4 of monkey striate cortex. The Journal of Neuroscience 15: 1808-1818, 1995.

Livingstone MS, Pack CC, and Born RT. Two-Dimensional Substructure of MT Receptive Fields. Neuron 30: 781-793, 2001.

Mayo JP, and Sommer MA. Neuronal Adaptation Caused by Sequential Visual Stimulation in the Frontal Eye Field. J Neurophysiol 100: 1923-1935, 2008.

Mayo JP, and Sommer MA. Neuronal correlates of visual time perception at brief timescales. Proceedings of the National Academy of Sciences 110: 1506-1511, 2013.

Mayo JP, and Sommer MA. Shifting attention to neurons. Trends Cogn Sci 14: 389, 2010. Mohler CW, Goldberg ME, and Wurtz RH. Visual receptive fields of frontal eye field neurons. Brain Res 61: 385-389, 1973.

Noudoost B, Chang MH, Steinmetz NA, and Moore T. Top-down control of visual attention. Curr Opin Neurobiol 20: 183-190, 2010. 
Pack CC, and Born RT. Temporal dynamics of a neural solution to the aperture problem in visual area MT of macaque brain. Nature 409: 1040-1042, 2001.

Park IM, Meister MLR, Huk AC, and Pillow JW. Encoding and decoding in parietal cortex during sensorimotor decision-making. Nat Neurosci 17: 1395-1403, 2014.

Phillips AN, and Segraves MA. Predictive Activity in Macaque Frontal Eye Field Neurons During Natural Scene Searching. 2010, p. 1238-1252.

Pillow JW, Shlens J, Paninski L, Sher A, Litke AM, Chichilnisky EJ, and Simoncelli EP. Spatio-temporal correlations and visual signalling in a complete neuronal population. Nature 454: 995-999, 2008.

Pouget P, Emeric EE, Stuphorn V, Reis K, and Schall JD. Chronometry of Visual Responses in Frontal Eye Field, Supplementary Eye Field, and Anterior Cingulate Cortex. J Neurophysiol 94: 2086-2092, 2005.

Reid CR, and Alonso J-M. Specificity of monosynaptic connections from thalamus to visual cortex. Nature 378: 281-284, 1995.

Rust NC, Schwartz O, Movshon JA, and Simoncelli EP. Spatiotemporal Elements of Macaque V1 Receptive Fields. Neuron 46: 945-956, 2005.

Sakai HM, and Naka K. Signal transmission in the catfish retina. V. Sensitivity and circuit. 1987, p. $1329-1350$.

Schall JD. On the role of frontal eye field in guiding attention and saccades. Vision Res 44: 1453-1467, 2004.

Schall JD, Morel A, King DJ, and Bullier J. Topography of visual cortex connections with frontal eye field in macaque: convergence and segregation of processing streams. J Neurosci 15: 4464-4487, 1995.

Schall JD, Sato TR, Thompson KG, Vaughn AA, and Juan C-H. Effects of Search Efficiency on Surround Suppression During Visual Selection in Frontal Eye Field. J Neurophysiol 91: 27652769, 2004.

Smith MA, Bair W, and Movshon JA. Dynamics of suppression in macaque primary visual cortex. J Neurosci 26: 4826-4834, 2006.

\section{Smith MA, Kelly RC, and Lee TS. Dynamics of Response to Perceptual Pop-Out Stimuli in} Macaque V1. 2007, p. 3436-3449.

Smith MA, Majaj NJ, and Movshon JA. Dynamics of motion signaling by neurons in macaque area MT. Nat Neurosci 8: 220-228, 2005.

Sommer MA, and Wurtz RH. Brain Circuits for the Internal Monitoring of Movements. Annu Rev Neurosci 31: 317-338, 2008. 
Sommer MA, and Wurtz RH. Influence of the thalamus on spatial visual processing in frontal cortex. Nature 444: 374-377, 2006.

590 Stanford TR, Shankar S, Massoglia DP, Costello MG, and Salinas E. Perceptual decision

591 making in less than 30 milliseconds. Nat Neurosci 13: 379-385, 2010.

592 Thompson KG, and Schall JD. The detection of visual signals by macaque frontal eye field

593 during masking. Nat Neurosci 2: 283-288, 1999.

594 Umeno MM, and Goldberg ME. Spatial processing in the monkey frontal eye field. I. Predictive 595 visual responses. J Neurophysiol 78: 1373-1383, 1997.

596 Womelsdorf T, Anton-Erxleben K, Pieper F, and Treue S. Dynamic shifts of visual receptive 597 fields in cortical area MT by spatial attention. Nat Neurosci 9: 1156-1160, 2006.

598 Wurtz RH. Neuronal mechanisms of visual stability. Vision Res 48: 2070-2089, 2008.

599 Xiao Q, Barborica A, and Ferrera VP. Radial motion bias in macaque frontal eye field. Vis 600 Neurosci 23: 49-60, 2006.

601 Yeh C-I, Xing D, Williams PE, and Shapley RM. Stimulus ensemble and cortical layer

602 determine V1 spatial receptive fields. Proceedings of the National Academy of Sciences 106:

603 14652-14657, 2009.

604 Zirnsak M, Steinmetz NA, Noudoost B, Xu KZ, and Moore T. Visual space is compressed in 605 prefrontal cortex before eye movements. Nature 507: 504-507, 2014.

606

607

608 
609

610

611

612

613

614

615

616

617

618

619

620

621

622

623

624

625

626

627

628

629

630

631

632

633

634

\section{Figure Legends}

Figure 1. Conventional and probabilistic ( $g l m R F)$ receptive field mapping for an example neuron. A, Polar plot illustrating direction tuning of the visual response (50-150 ms after target onset) during the memory-guided saccade task. The black triangle indicates the vector fit of the preferred direction. B, Tuning of the visual response at six eccentricities along the preferred direction. C, Schematic illustrating the GLM model; see Methods for details. D, Resulting glmRF for this neuron.

Figure 2. A, Three additional example glmRFs, with an arrow indicating the direction and amplitude of the preferred visual stimulus using conventional RF mapping. Thin, dashed black lines outline the significant contiguous pixels as determined by permutation test. B, Distribution of angular differences between the preferred direction from conventional RF mapping and the peak of the glmRF (from a 2D Gaussian fit). C, Scatter plot of the amplitude or eccentricity of the RF as determined by conventional RF mapping and the glmRF. Note that the extra data point in this panel represents a neuron for which we measured amplitude but not direction tuning. D, Ellipsoids representing the 2D Gaussian fit are plotted here for all neurons with a significant glmRF. Each ellipse is drawn with its major and minor axis radius equal to one standard deviation of the best fit Gaussian's corresponding axis. Red represents neurons recorded from the left hemisphere, blue represents neurons recorded from the right hemisphere for the glmRFs for neurons that passed the cross-validation test (solids lines) or that failed the cross-validation but passed the resampling test (dashed lines). E, Scatter plot of the RF area as a function of RF eccentricity for the glmRFs for neurons that passed the cross-validation test (black dots and line) or that failed the cross-validation but passed the resampling test (grey dots and line). 
637 Figure 3. A, Spatial plots of glmRFs over time for three example neurons with 30 ms image 638 duration (left column) and $10 \mathrm{~ms}$ image duration (middle and right columns). In all three panels, 639 the ticks on the color bars represent a step of 0.2 in the $\beta$ value of the GLM, and the vertical and 640 horizontal ticks on each frame represent steps of $5^{\circ}$ as in Figures 1D and 2A. 10 ms frames in 641 the middle and right columns are stacked so that each row, across columns represents a $30 \mathrm{~ms}$ 642 progression in time over a total latency range of $270 \mathrm{~ms}$. The blue stars indicate the optimal 643 window delay and width for each of the three neurons. B, Temporal kernels for the same three neurons showing the spatial variance at each temporal offset. C, Matrices of the r-values of the 645 GLM at each window delay and window width tested.

Figure 4. A, Population-averaged temporal kernels for the neurons tested at an image duration 649 of $30 \mathrm{~ms}$ (blue line) and $10 \mathrm{~ms}$ (red line). The shading indicates \pm 1 SEM. B. Average of the $r$ value matrices for all neurons at the optimal condition for each neuron $(n=73)$.

653 Figure 5. Spatiotemporal tuning by task. A-C, Example glmRFs for three neurons (one for each 654 task). D, Image duration task, where a dot was held on the screen for 30,70 or 150 ms before a 655 new dot location was chosen. E, Image duration task, with each dot held on the screen for 10 or $65670 \mathrm{~ms}$. Note that one outlying neuron was removed for display purposes (although it followed 657 the same trend). F, Dot density task, with image duration held at $70 \mathrm{~ms}$ while the number of dots 658 per image was 1, 2 or 8. Dashed lines represent cells that failed the cross-validation test. Each 659 panel (D-F) shows the normalized mean beta for each of the recorded neurons (gray lines) in all 
660 conditions, with the mean across neurons shown by a thick black line. Red lines represent 661 example neurons. 

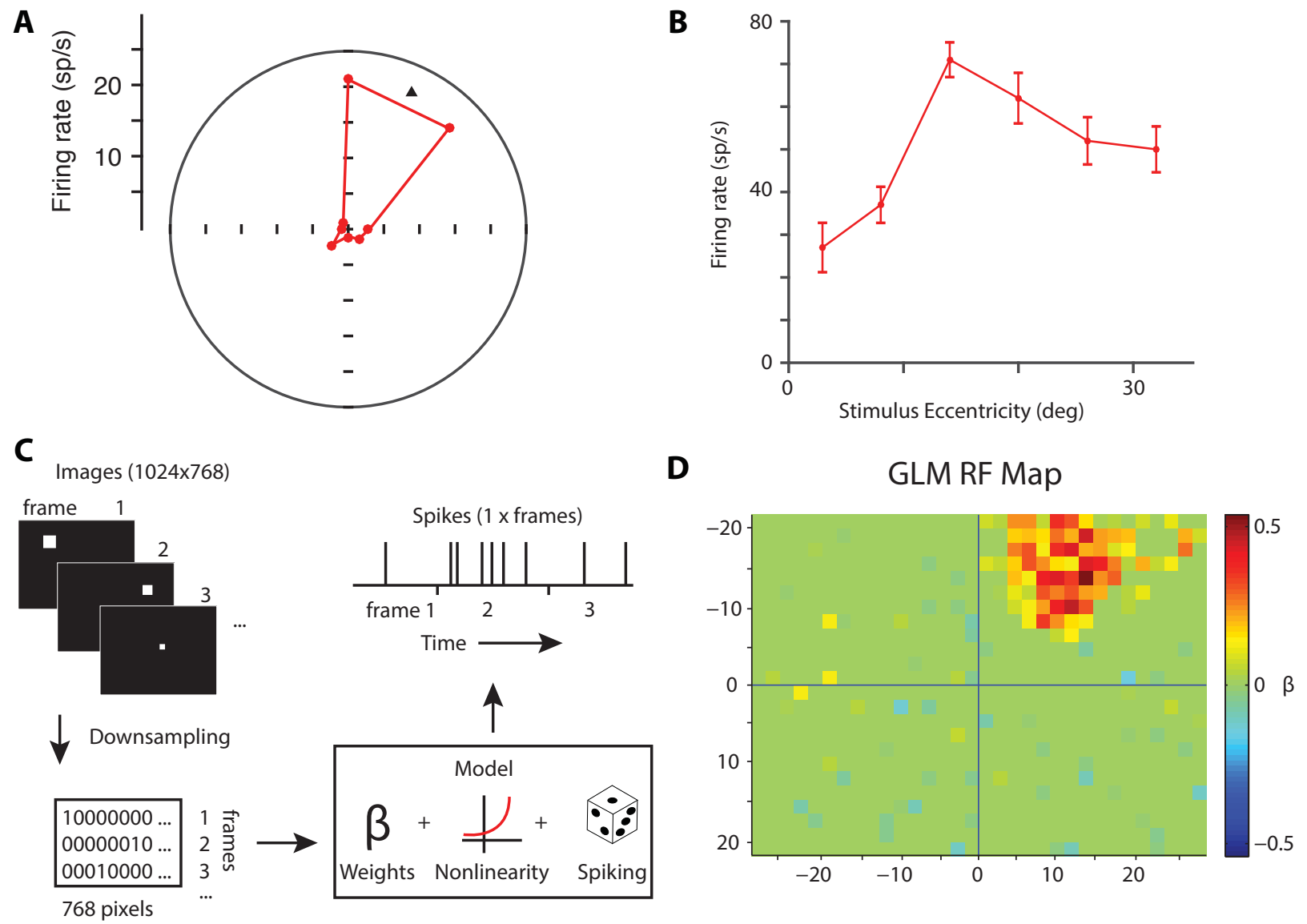


\section{A}
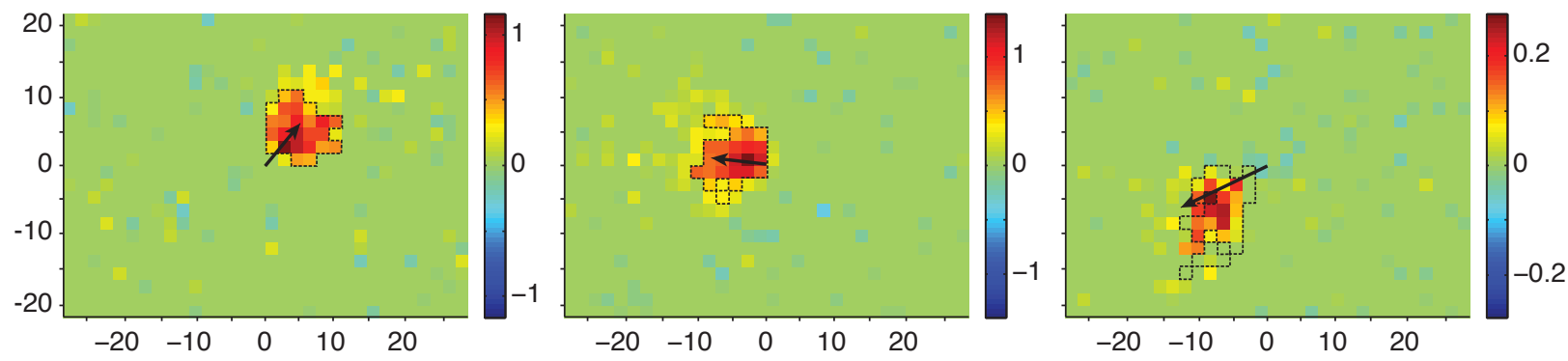

B

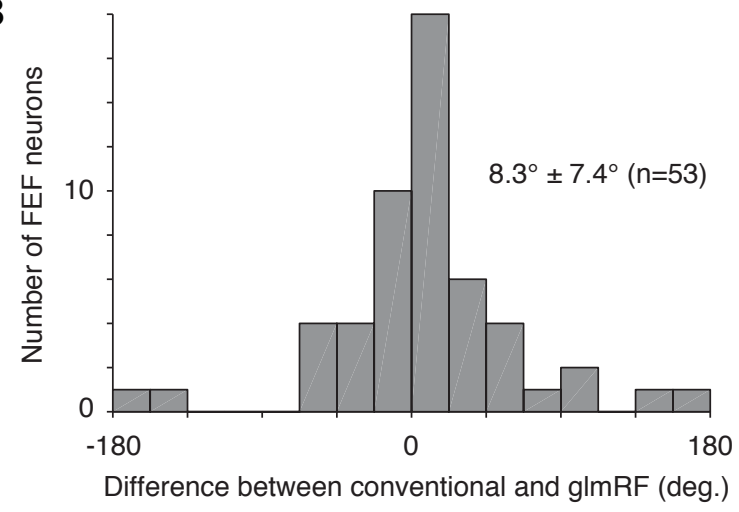

D

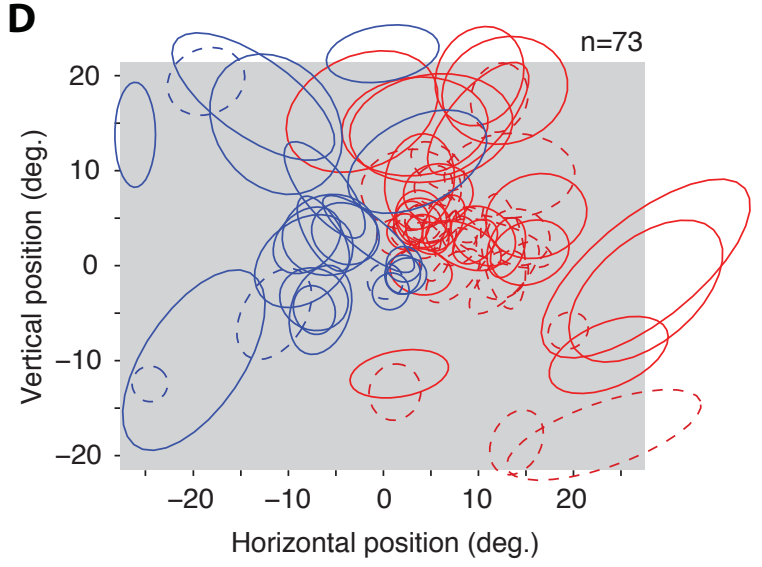

C

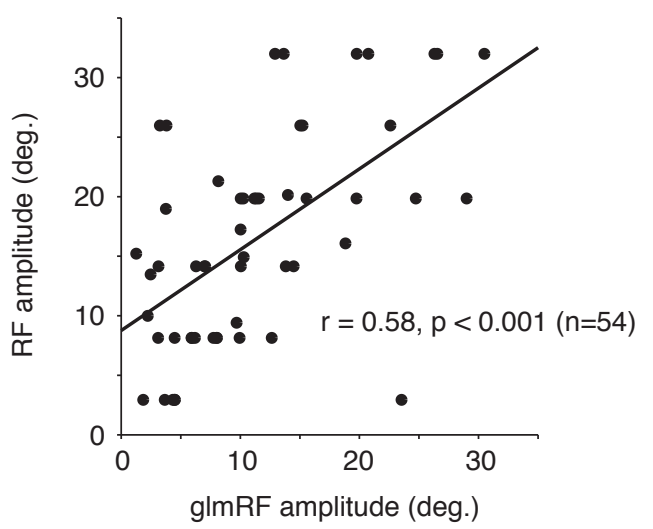

E

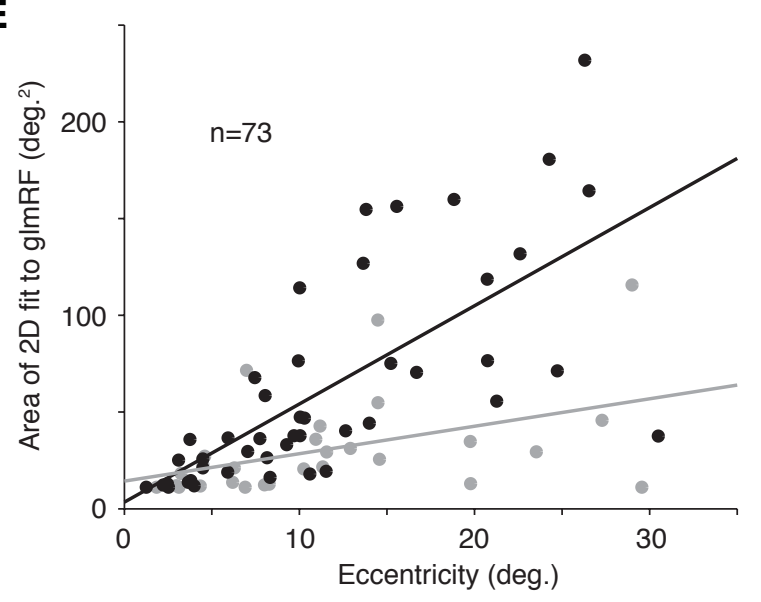




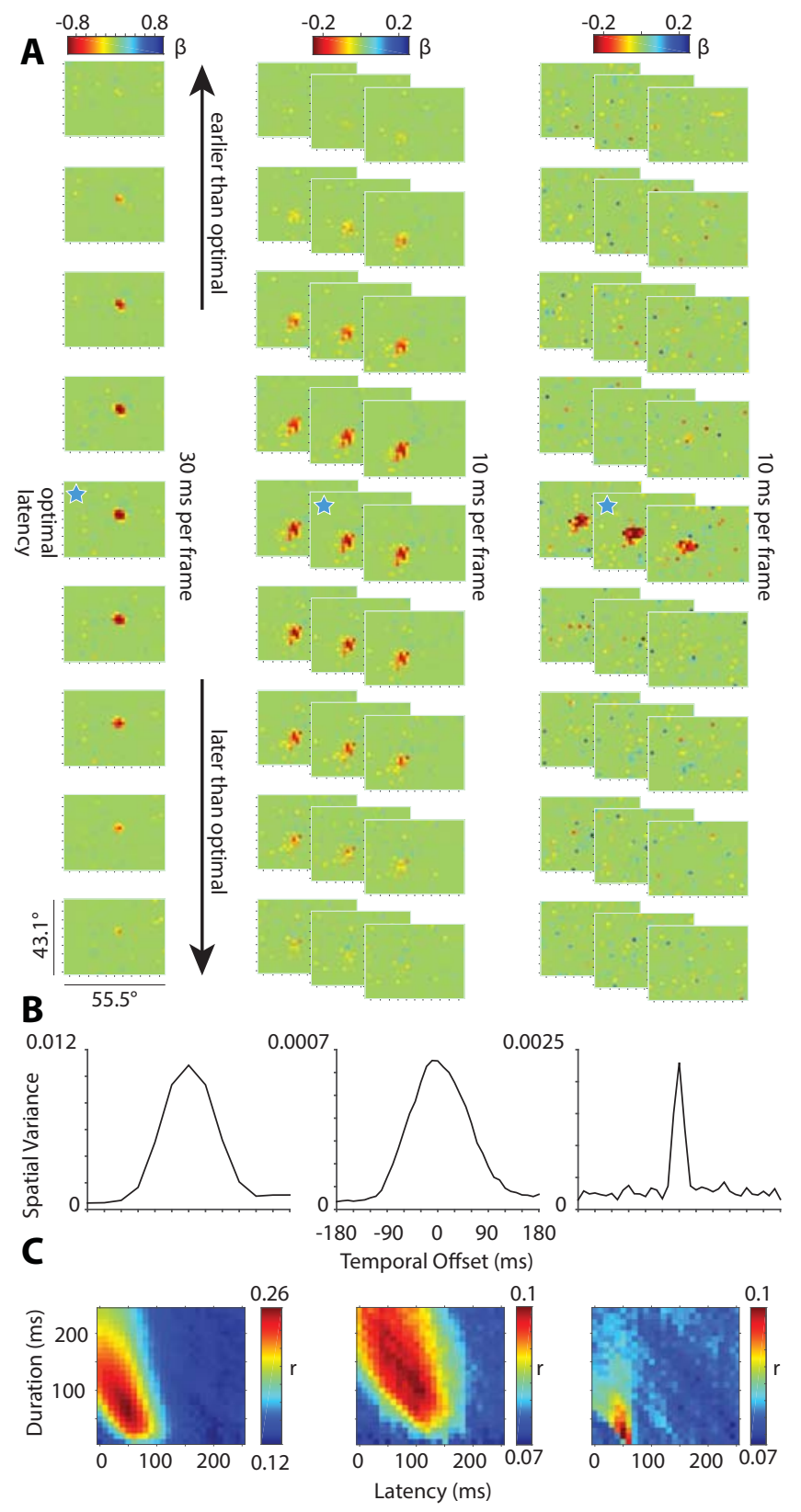




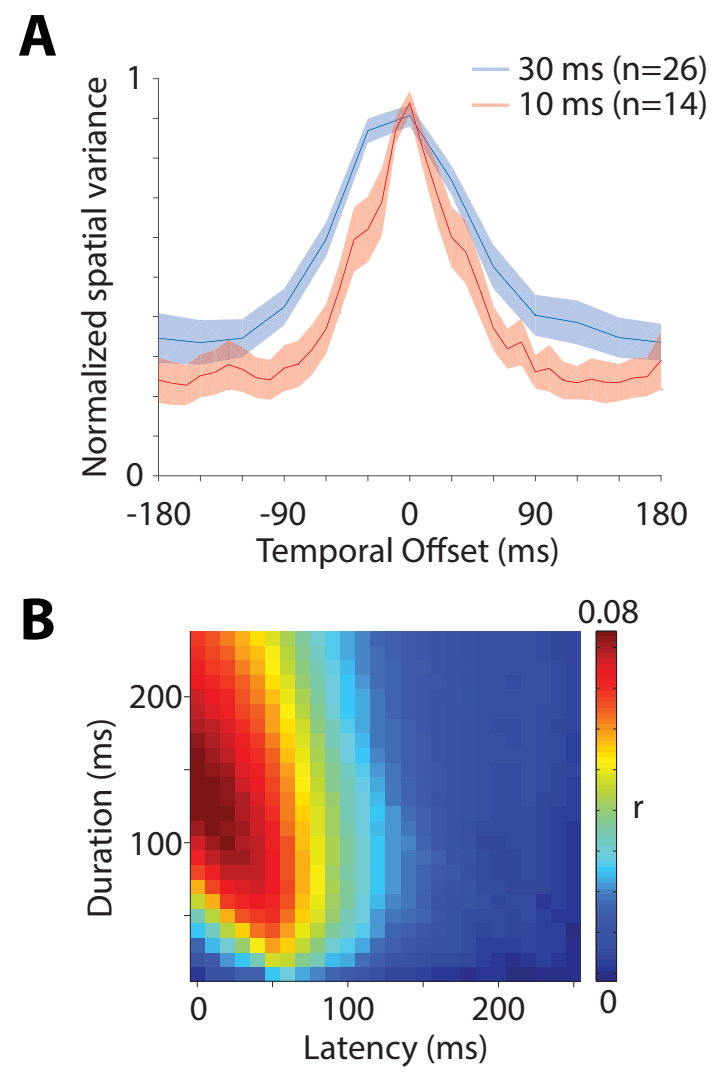




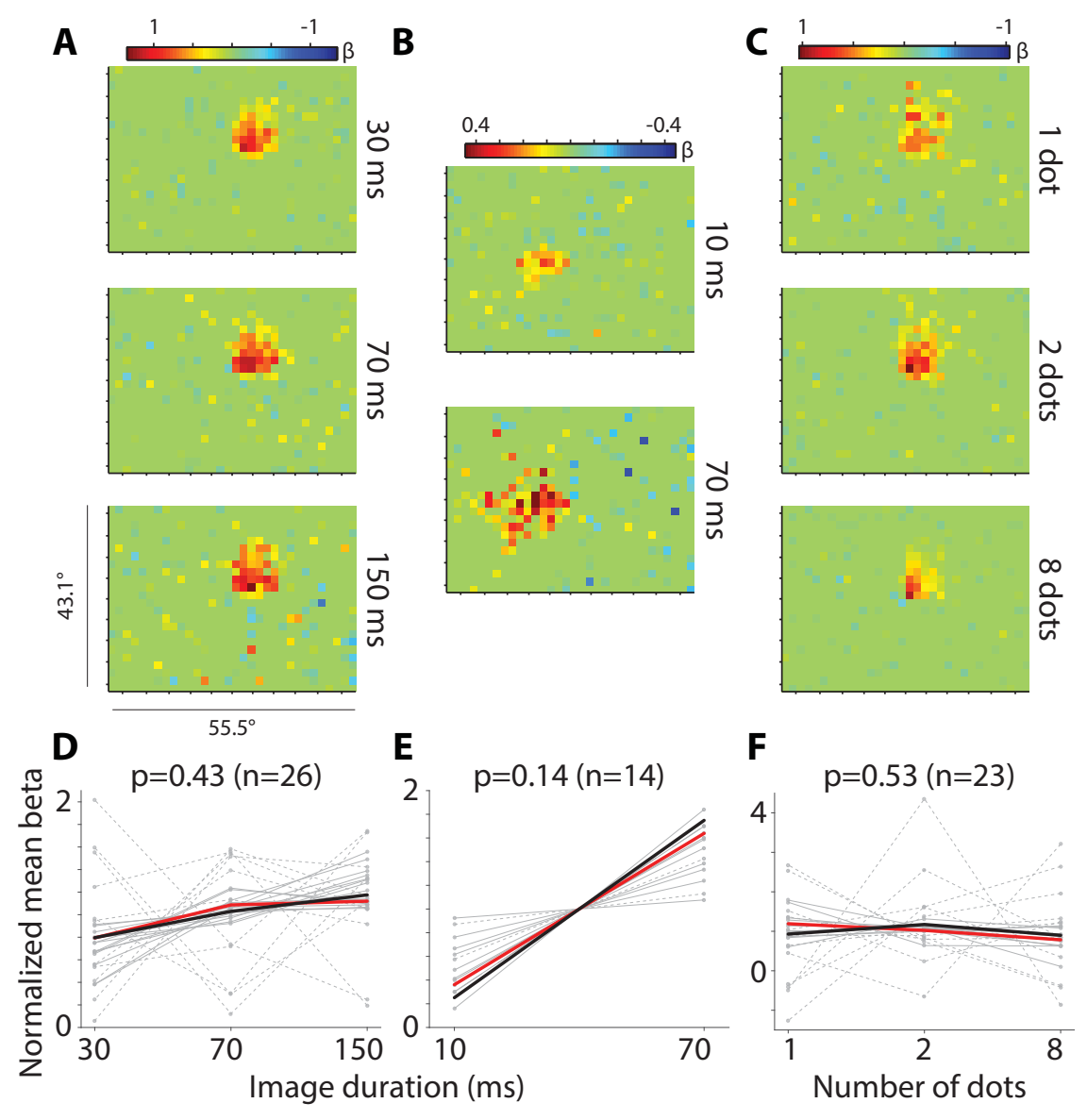

